Bio - grafía. Escritos sobre la Biología y su Enseñanza. ISSN 2027-1034

Edición Extraordinaria. p.p. $799-807$

Memorias del IX Encuentro Nacional de Experiencias en Enseñanza de la Biología y la Educación Ambiental. IV Congreso Nacional de Investigación en Enseñanza de la Biología.

\title{
APRENDIZAJES CONCEPTUALES ALCANZADOS POR LOS ESTUDIANTES DE TRES ESCUELAS RURALES EN CHIPATÁ - SANTANDER EN RELACIÓN ALA ECOLOGÍA Y CONSERVACIÓN DE LAS AVES
}

\section{CONCEPTUAL LEARNING REACHED BY THE STUDENTS FROM THREE RURAL SCHOOLS IN CHIPAT Á-SANT ANDER REGARDING THE ECOLOGY AND BIRDS CONSERVATION}

\author{
Martínez Parra, Miguel Ángel ${ }^{1.2}$ \\ Cepeda Benavides, Wilson ${ }^{1.3}$
}

\section{RESUMEN}

En este trabajo se divulgan los resultados parciales del proyecto "contribución a la construcción del concepto de «Conservación de Aves` por parte de los estudiantes de tres sedes rurales del Instituto Técnico Agropecuario "Agatá" en el municipio de ChipatáSantander" ; donde se vinculan la escuela rural y la enseñanza-aprendizaje de las ciencias para la conservación de la biodiversidad. Se implementó una unidad didáctica bajo el enfoque de la investigación escolar. Los aprendizajes conceptuales logrados fueron analizados mediante categorías; se encontró que estos contribuyen a la construcción del concepto de conservación de aves enfocado a su función de mantenimiento y equilibrio en el ecosistema.

PALABRAS CLAVES: Aprendizajes Conceptuales, Conservación de aves, Contexto rural.

\section{ABSTRACT}

This article shows partial results of the project "Contribution to the construction of the «Bird Consevation> concept by students from three rural branches of the Instituto Técnico Agropecuario "Agatá" (school) in Chipatá township in Santander", where rural school and teaching-learning of science are articulated towards biodiversity conservation. A didactic unit was implemented according to the scholar research approach. The gained conceptual learnings were analyzed through categories and it was found that those learnings contribute to the construction of «Bird Conservation) concept applied to their role on the maintenance and balance of the ecosystem.

1. Estudiante Proyecto Curricular Licenciatura En Biología Grupo de investigación Biología, Enseñanzay Realidades BER. Univers idad Distrital Francisco José de Caldas.

2. Correo: miamartíinezp@correo.udistrital.edu.co

3. Correo: wcepedab@correo.udistrital.edu.co 
Bio - grafía. Escritos sobre la Biología y su Enseñanza. ISSN 2027-1034

Edición Extraordinaria. p.p. $799-807$

Memorias del IX Encuentro Nacional de Experiencias en Enseñanza de la Biología y la

Educación Ambiental. IV Congreso Nacional de Investigación en Enseñanza de la Biología.

KEY WORDS: Conceptual learnings, Birds conservation, Rural context.

\section{INTRODUCCIÓN}

La conservación de una o varias especies es un campo complejo debido a los múltiples factores con los que se relaciona, por esta razón la solución a estas problemáticas no está solamente en las manos de las instituciones educativas, sino que también depende, en gran medida, de la población humana que en su legado cultural y sus costumbres intervienen conscientemente o no, en la conservación o el deterioro de las especies de búhos. Para tal cometido se espera dar una nueva perspectiva a los modelos de enseñanza (Cañal P. , 2004): se les debe promover a los estudiantes problemáticas socio-ambientales donde los conceptos biológicos sean involucrados. En el campo de la didáctica de la biología, este trabajo se enfatiza en los procesos de enseñanza y aprendizaje de los conceptos biológicos en este caso, el de la conservación de una o varias especies de búhos familia Strigidae, colibríes familia Trochilidae, guacharacas familia Cracidae y toches familia Ictiridae, es de gran importancia para este campo de la ciencia la puesta en marcha del trabajo en un lugar donde los aportes de la didáctica de la biología son escasos, lo que posiblemente abrirá las puertas a muchas investigaciones en el desarrollo de este mismo concepto o en otros conceptos para estas especies o para las que conforman el contexto de los estudiantes. Así se logrará que los estudiantes comprendan la importancia de cuidar y conservar estas aves en su contexto como parte vital de un ecosistema natural.

\section{METODOLOGÍA}

Las tres sedes educativas: el Rocío, el Papayo y Puente Grande, pertenecen al Instituto Técnico Agropecuario "Agatá" del municipio de Chipatá, Santander. El estudio se realizó con 27 estudiantes de edades entre los 9 y 14 años, en los cursos $4^{\circ}$ y $5^{\circ}$ primaria. A los cuales se le hizo el seguimiento a la mitad del grupo. $(n=14)$ teniendo como criterio el nivel de aprendizaje logrados. Se diseñaron 17 sesiones de clase de una duración de cada una entre 2 a 5 horas durante 5 meses con enfoque investigativo, relacionados con una unidad didáctica donde las 17 sesiones se dividían en cuatro fases: la fase 1 denominada: conociéndonos; la fase 2: preparándome como investigador; la fase 3: investigando y finalmente la fase 4: compartiendo mis aprendizajes. Para los análisis de los resultados en cada una de las sesiones se realizó una un análisis mediante categorías teniendo en cuenta los instrumentos de recolección de datos: diario de campo tanto del estudiante como del profesor, guías de campo, grabaciones en audio y video e ilustraciones por los estudiantes. Con el propósito de enmascarar la información correspondiente a los nombres de los estudiantes participantes se les designó una referencia alfanumérica a cada uno, según la escuela a la que pertenecen. (ver Tabla 1). 
Bio - grafía. Escritos sobre la Biología y su Enseñanza. ISSN 2027-1034

Edición Extraordinaria. p.p. 799 - 807

Memorias del IX Encuentro Nacional de Experiencias en Enseñanza de la Biología y la Educación Ambiental. IV Congreso Nacional de Investigación en Enseñanza de la Biología.

Tabla 1. Designación de los estudiantes para el análisis de los datos

\begin{tabular}{|c|c|c|c|c|c|}
\hline \multicolumn{6}{|c|}{ ESCUELAS } \\
\hline \multicolumn{2}{|c|}{ EL ROCío } & \multicolumn{2}{|c|}{ EL PAPAYO } & \multicolumn{2}{|c|}{ PUENTE GRANDE } \\
\hline R1 & R2 & P1 & P2 & PG1 & PG2 \\
\hline R3 & R4 & P3 & P4 & PG3 & PG4 \\
\hline R5 & R6 & P5 & P6 & PG5 & PG6 \\
\hline R7 & R8 & P7 & P8 & PG7 & \\
\hline & & P9 & P10 & & \\
\hline & & P11 & P12 & & \\
\hline
\end{tabular}

\section{RESULT ADOS Y DISCUSIÓN}

\section{Categorización aprendizajes conceptuales.}

A continuación, se tabulan los resultados de los casos por las tres escuelas recogidos en todas las sesiones de clase para el análisis de la categorización de los aprendizajes conceptuales (ver Tabla 2)

- Subcategoría Ecología de las aves.

En subcategoría se aborda la ecología de las aves desde su papel en el ecosistema, así, los estudiantes nombran algunas relaciones que tienen las aves con otras aves, con otros animales, con las plantas, con los humanos y con el entorno, en un nivel mayor, abarca relaciones más complejas entre las aves y las plantas principalmente con los procesos de alimentación y dispersión de semillas y polinizadores, (Moreno, 2010) como proceso importante e determinan la estructura espacial de las poblaciones de plantas, además, reconoce la diferencia entre cadena y red trófica (Cepeda, Martínez, \& Rangel, 2015)

Tabla 2. Aprendizajes conceptuales categorizados.

\section{APRENDIZAJES CONCEPTUALES}

\begin{tabular}{|c|c|c|}
\hline \multirow[t]{2}{*}{$\begin{array}{c}\text { CATEGORÍ } \\
\text { A } \\
\text { AVES }\end{array}$} & \multirow[t]{2}{*}{$\begin{array}{c}\text { SUBCATEGORÍA } \\
\mathbf{S}\end{array}$} & $\begin{array}{l}\text { CASOS POR SEDES REGISTRADOS EN: } \\
\text { (DIARIO DEL PROFESOR, DIARIOS DE CAMPO } \\
\text { DEL ESTUDIANTE, CUADERNO DE LOS } \\
\text { ESTUDIANTE, GRABACIONES AUDITIVAS Y/O } \\
\text { VISUALS, ESCRTOS O DOCUMENTOS } \\
\text { REALIZADOS POR LOS ESTUDIANTES O I } \\
\text { PROFESORES, FORMATOS DE REGISTRO EN } \\
\text { RED/ VISUAL E ILUSTRACIONES POR LOS } \\
\text { ESTUDIANTES) }\end{array}$ \\
\hline & & $\begin{array}{c}\text { APRENDIZAJE } \\
\text { MOMENTO FINAL }\end{array}$ \\
\hline
\end{tabular}


Bio - grafía. Escritos sobre la Biología y su Enseñanza. ISSN 2027-1034

Edición Extraordinaria. p.p. $799-807$

Memorias del IX Encuentro Nacional de Experiencias en Enseñanza de la Biología y la Educación Ambiental. IV Congreso Nacional de Investigación en Enseñanza de la Biología.

ROCIO

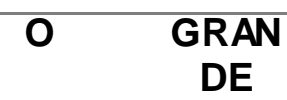

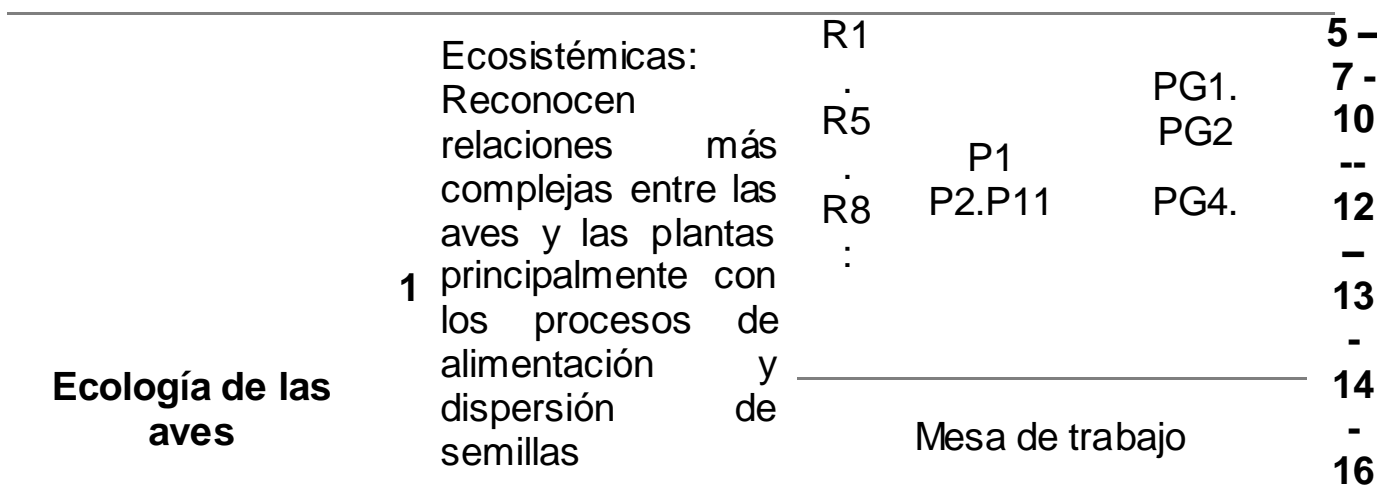

Conservación

\begin{tabular}{|c|c|c|c|c|}
\hline $\begin{array}{l}\text { Reconoce las } \\
\text { interacciones que } \\
\text { suceden dentro del } \\
\text { ecosistema, } \\
\text { además, incluye, } \\
\text { las relaciones, } \\
2 \text { sociales culturales, } \\
\text { políticas y } \\
\text { adicional, los } \\
\text { procesos que } \\
\text { conllevan a perder, } \\
\text { mantener o crear } \\
\text { esas relaciones }\end{array}$ & & \multicolumn{2}{|c|}{ En el documento final } & 16 \\
\hline $\begin{array}{l}\text { Reconoce el papel } \\
\text { de las aves dentro } \\
\text { del ecosistema } \\
\text { como } \\
1 \text { fundamentales en } \\
\text { los procesos de } \\
\text { conservación }\end{array}$ & $\begin{array}{l}\mathrm{R} 1 \\
\text { R5 } \\
\text { R6 } \\
\text { R8 }\end{array}$ & $\begin{array}{l}\text { P1. P2. } \\
\text { P9. } \\
\text { P11. P6, } \\
\text { P7, P9. }\end{array}$ & $\begin{array}{l}\text { PG2. } \\
\text { PG3. } \\
\text { PG4. } \\
\text { PG7. }\end{array}$ & $\begin{array}{l}5- \\
14\end{array}$ \\
\hline $\begin{array}{l}\text { Identifica las aves } \\
\text { como: } \\
2 \text { bioindicadores y } \\
\text { controladores } \\
\text { biológicos, }\end{array}$ & $\begin{array}{l}\text { R1 } \\
\text { R5 } \\
\text { R8 }\end{array}$ & $\begin{array}{l}\text { P2. P7. } \\
\text { P9. } \\
\text { P11. }\end{array}$ & $\begin{array}{l}\text { PG1. } \\
\text { PG2. } \\
\text { PG4. } \\
\text { PG7. }\end{array}$ & 14 \\
\hline
\end{tabular}


Bio - grafía. Escritos sobre la Biología y su Enseñanza. ISSN 2027-1034

Edición Extraordinaria. p.p. $799-807$

Memorias del IX Encuentro Nacional de Experiencias en Enseñanza de la Biología y la Educación Ambiental. IV Congreso Nacional de Investigación en Enseñanza de la Biología.

Mesas

En el documento final elaborado por los estudiantes de las tres escuelas

En esta categoría de mayor complejidad, el estudiante sintetiza todas las relaciones del ave, con su entorno incluyendo el humano, y todas las relaciones que tiene el humano incluyendo las aves, en ésta los estudiantes complejizan algunas de ellas principalmente las que analizaron en su investigación, en el documento final los estudiantes de las tres escuelas recogen sus aportes: en cuanto a las interacciones: Las aves hacen crecer plantas" "La importancia de las aves para nosotros es, que nos ayudan a esparcir las semillas, para que crezcan más árboles, son importantes para el ecosistema, comiéndose algunos animales como el búho como los ratones, y ayuda con el equilibrio de la naturaleza (P2) en cuanto a las relaciones político-sociales, culturales y la pérdida o creación de las relaciones y las interacciones: Algunas actividades que representan un beneficio para el hombre en la actualidad pueden afectar a los ecosistemas, ... la tala de árboles, ... la contaminación de la naturaleza por la fumigación de las plantas, que contribuye a que muchos animales se vean afectados ... podemos cambiar esta situación intentando encontrar un equilibrio entre el ecosistema y el hombre, donde podamos seguir con nuestras actividades pero sin deteriorar nuestro medio ambiente, ya que si afectamos este se vería afectada nuestra relación con todo nuestro entorno y se vería peligrada nuestra existencia.

...La consenación nos enseña a respetar la naturaleza, manteniendo un beneficio para nosotros sin tener que afectar la naturaleza y logramos esto podemos permitir que haya un mejor mañana para todos con más árboles, sin contaminación y con el cual podríamos apreciar los hermosos paisajes, animales y plantas que nos brinda la naturaleza. (Fuente diario de campo del estudiante y del profesor, grabaciones de sonido y video, Documento Final), es así que los estudiantes integran de una manera más compleja los procesos ecológicos que se dan en su contexto, trascienden sus ideas previas de que las aves solo están como adorno o para cantarnos y exploran un sin fin de relaciones e interacciones que tienen este grupo, aclaran el concepto de conservación, reconocen las causas y los efectos de la actividad humana y brindan herramientas para equilibrar el ecosistema de manera sostenible, así el aporte que hacen los estudiantes a este estudia va de paralelo a las recomendaciones hechas por . (Rozzi, et al. 2005, pág. 23) Donde sustenta que: la crisis de la conservación de la diversidad biológica y cultural es en gran medida una crisis cognitiva y vivencial porque no percibimos a la mayoría de los seres y sus procesos vitales; todavía menos percibimos la integralidad de la vida de cada ser vivo si no convivimos con ellos. (Rozzi, et al. 2005 (pág. 23) 
Bio - grafía. Escritos sobre la Biología y su Enseñanza. ISSN 2027-1034

Edición Extraordinaria. p.p. $799-807$

Memorias del IX Encuentro Nacional de Experiencias en Enseñanza de la Biología y la

Educación Ambiental. IV Congreso Nacional de Investigación en Enseñanza de la

Biología.

Los estudiantes insisten en que se debe existir un equilibrio en el ecosistema, seguido de un proceso de recuperación, en el cual no sólo se reconozcan los entes perturbadores sino que se contribuya a la solución de problemáticas detectadas en el entorno, así el proceso de conservación será efectivo ya que es significativo para los que al terminar el estudio permanezcan en el territorio, con una nueva idea de conservación no solo de las aves sino del ambiente. Este trabajo apoya la idea de que: Los resultados de la investigación en este estudio debe basarse en corroborar que la naturaleza debe ser colocado en el corazón de la educación escolar de los niños. (Richardson, Sheffield, Harvey, \& Petronzi, 2016, pág. 21).

\section{- Subcategoría Conservación de las aves}

En esta subcategoría se entiende la conservación de las aves como una disciplina en donde se puede contribuir a la permanencia de las especies en el territorio, donde se entiende esta conservación como una mediación entre el campo disciplinar y otras disciplinas de orden social que puedan dinamizar su campo de acción, además relaciona la comunidad estos procesos.La mayoría de los estudiantes comprenden el concepto de conservación de aves como proceso fundamental en el mantenimiento ecosistémico reconociendo que las aves siembran árboles y siven de alimento para otros animales, $y$ desequilibraría la cadena alimenticia y al ecosistema (R8); La importancia de las aves para nosotros es, que nos ayudan a esparcir las semillas, para que crezcan más árboles, son importantes para el ecosistema, comiéndose algunos animales como el búho como los ratones, y ayuda con el equilibrio de la naturaleza (P2); algunos riegan semillas por los bosques y algunos como el colibrí polinizan a las plantas o el chulo come carroña o el cadillero (Generos: Astragalinus y Sporagra ) come cadillo o pegapega (Fabaceae) (PG2), inclusive entienden el concepto de conservación como cuidar la naturaleza, el ecosistema para nuestro beneficio y el de las aves (R5); podemos decir que la conservación es una forma en la cual podemos encontrar un equilibrio entre la naturaleza y las actividades que son realizadas por el hombre, donde los dos se pueden ver beneficiados (Documento Final). (Fuente diario de campo del estudiante y del profesor, grabaciones de sonido y video, Documento Final), se resalta que entendiendo el papel fundamental del ave en el ecosistema inevitablemente entienden el concepto de conservación, ya que si relacionan las aves con la dispersión de semillas, donde más hacen hincapié, esa dispersión beneficia directa o indirectamente al humano.

En cuanto bioindicadores y controladores biológicos los estudiantes argumentan: Entendiendo esta importancia sabemos cómo nos pueden beneficiar las aves, ya que comprendemos que relaciones podemos tener con ellas y ellas con nosotros, $y$ a partir de esto podemos decidir de qué manera podemos contribuir al beneficio de nuestros ecosistemas entendiendo que nos podemos beneficiar al tiempo que ayudamos a que se mantengan nuestros ecosistemas (Documento final);estos animales (colibríes) riegan semillas que dan origen a otras gracias a que toman el néctar de las flores y los depositan en diferentes partes donde se encuentran las flores... (Mesa de trabajo Nectarívoros); el búho ayuda con los roedores y se les prende polen y ayudan a trasportar semillas y si el búho muere aumenta los roedores.(PG4); Las aves ayudan a controlar plagas (Ratones, Insectos), comen carroña, dispersan semillas, nos brindan alimento, ayudan a abonar y nos relacionamos con ellas cuidando el medio ambiente(P6, P7, P9) 
Bio - grafía. Escritos sobre la Biología y su Enseñanza. ISSN 2027-1034

Edición Extraordinaria. p.p. $799-807$

Memorias del IX Encuentro Nacional de Experiencias en Enseñanza de la Biología y la

Educación Ambiental. IV Congreso Nacional de Investigación en Enseñanza de la

Biología.

(Fuente diario de campo del estudiante y del profesor, grabaciones de sonido y video, Documento Final) Con estos argumentos los estudiantes comprenden que las aves aportan beneficios no solo al humano sino también al ecosistema, controlando plagas, y primordialmente aumentando las posibilidades de que las plantas se reproduzcan, (mediante la polinización), se dispersen (llevándolas en su pico o en su aparato digestivo) y se desarrollen (por procesos de digestión interna y depósito de material orgánico o popó como lo argumentan los estudiantes).

\section{CONCLUSIONES}

Se evidenció que los estudiantes establecen relaciones cognitivas complejas con respecto a las relaciones ecosistémicas que tienen las aves, principalmente en el rol de dispersores de semillas, controladores biológicos y en el mantenimiento del equilibrio ecosistémico que ocupe, por ende, la necesaria investigación para la conservación del grupo.

Los estudiantes coinciden en que conservación de las diferentes especies, no sólo de las aves, en un ecosistema debe regirse a partir de la investigación para el conocimiento y manejo adecuado de las relaciones inter e intraespecíficas, con el objeto de proponer e implementar acciones concretas en torno a las actividades que generen afectación.

En cuanto al concepto de conservación los estudiantes lo apropian como un beneficio conjunto entre aves y humano, a pesar de su corta edad todos los estudiantes comprenden este concepto de manera más compleja y contextual, primando el entendimiento y el mantenimiento de las relaciones ecosistémicas con las actividades antropogénicas.

\section{BIBLIOGRAFÍA}

Acevedo, J. (2004). Reflexiones sobre las finalidades de la enseñanza de las ciencias: educación científica para la ciudadanía. Revista Eureka sobre Enseñanza y Divulgación de las Ciencias, 1(1), 3-15.

Allen, M. (2015). Preschool Children's Taxonomic Knowledge of Animal Species. Journal of Research in Science Teaching, 52(1), 107-134. doi:10.1002/tea.21191

Alves, T., Carniel, P., \& Langguth, A. (2013). CAPTURA E COMERCIALIZAÇÃO DE ANIMAIS SILVESTRES NO SEMIÁRIDO DA PARAIBA, BRASIL, SOB A PERSPECTIVA DE CRIANÇAS E ADOLESCENTES. Revista Nordestina de Biología, 21(2), 79-100. 
Bio - grafía. Escritos sobre la Biología y su Enseñanza. ISSN 2027-1034

Edición Extraordinaria. p.p. $799-807$

Memorias del IX Encuentro Nacional de Experiencias en Enseñanza de la Biología y la

Educación Ambiental. IV Congreso Nacional de Investigación en Enseñanza de la Biología.

Angulo, A. (2011). "Dispersión de semillas" por aves frugívoras: una revisión de estudio de la región neotropical (Tesis). Bogotá: Pontificia Universidad Javeriana.

Braund, M. (1991). Children's ideas in classifying animals. Journal of Biological Education, 25(2), 10-110. doi:10.1080/00219266.1991.9655186

Caamaño, A. (2003). Los trabajos prácticos en ciencia. En M. Jiménez, A. Caamaño, A. Oñorbe, E. Pedrinaci, \& A. De Pro, Enseñar ciencias (págs. 95-118). Barcelona: Graó.

Cancino, U. S. (2001). El trabajo en equipo en la escuela. Revista Profissão Docente, 1(3), 1-14.

Cañal, P. (2004). La enseñanza de la Biología ¿Cuál es la situación actual y qué hacer para mejorarla? Revista Alambique didáctica de las ciencias experimentales, 2741.

Cepeda, W., Martínez, M., \& Rangel, M. (2015). "COMPRENSIÓN DEL CONCEPTO DE RED TRÓFICA Y SU DIFERENCIA CON CADENA TRÓFICA MEDIANTE TRABAJOS PRÁCTICOS Y PREGUNTAS CONFLICTO PARA TRES ECOSISTEMAS COLOMBIANOS". Bio-grafia, extraordinario, 1712 - 1720.

Dixon, S. P., Birchenough, A. C., Evans, S. M., \& Quigley, M. P. (2005). Children's knowledge of birds: How can it be improved and can it be used to conserve wildlife. Transactions of the Natural History Society, Northumbria, 64, 121-134.

Furió, C., \& Furió, C. (2000). Dificultades conceptuales y epistemológicas en el aprendizaje de los procesos químicos. Educación Química, 11(3), 300-308.

Howe, D., Kahn, P., \& Friedman, B. (1996). Along the Rio Negro: Brazilian Children's Environmental Views and Values. Developmental Psychology, 32(6), 979-987.

Izquierdo, M., Espinet, M., Bonil, J., \& Pujol, R. (2004). Ciencia escolar y complejidad. Investigación en la escuela, 53, 21-30.

Keb, M., \& Cervera, M. (2014). ¡VAMOS A PESCAR!: LOS NIÑOS MAYAS Y LAS AVES DE YUCATÁN, MÉXICO. En M. A. Vásquez, Aves, personas y culturas. Estudios de Etno-ornitología 1 (págs. 19-34.). Oaxaca,, México: CONACYT/ ITVO/Carteles Editores/UTCH.

Kellert, S. (1985). Attitudes toward animals: Age-related development among children. Journal of Environmental Education, 16(3), 29-39.

Kubiatko, M., Usak, M., \& Pecusova, E. (2011). Elementary school pupils knowledge and misconceptions about birds. Eurasian Journal of Educational Research, 43, 163181.

Moreira, M. A., \& Greca, I. M. (2003). Cambio conceptual : análisis crítico y propuestas a la luz de la teoría del aprendizaje significativo. Ciência \& Educação, 9(2), 301-315. 
Bio - grafía. Escritos sobre la Biología y su Enseñanza. ISSN 2027-1034

Edición Extraordinaria. p.p. $799-807$

Memorias del IX Encuentro Nacional de Experiencias en Enseñanza de la Biología y la

Educación Ambiental. IV Congreso Nacional de Investigación en Enseñanza de la Biología.

Moreno, S. (2010). Aves dispersoras de semillas en un remanente de bosque seco tropical en la finca Betanci-Gucamayas (Córdoba). Bogotá: Universidad Javeriana.

Muñoz, A. (2014). Aves rapaces y control bilógico de plagas. En A. Muñoz, j. Rau, \& j. Yáñez, Aves Rapaces de Chile (pág. 386). Valdivia: Aves Rapaces de Chile.

Parra, E. (2014). Aves silvestres como bioindicadores de contaminación ambiental y metales pesados. Revista CES Salud Pública, 5, 59-69.

Perrenoud, P. (2005). Diez nuevas competencias para enseñar. Barcelona: Graó.

Peters, V., Mordecai, R., Carroll, C., C. R., \& Greenberg, R. (2010). Bird community response to fruit energy. Journal of animal ecology, 79, 824-835. doi:10.1111/j.1365-2656.2010.01699.x

Pozo, J. (1999). Más allá del cambio conceptual: el aprendizaje de la ciencia como cambio representacional. Enseñanza de las Ciencias, 17(3), 513-520.

Pozo, J., del Puy, M., Angeles, S., \& Limón, M. (1991). Las ideas de los alumnos sobre ciencia como teorías implícitas. infancia y Aprendizaje, 57(3), 3-22.

Richardson, R., Sheffield, D., Harvey, H., \& Petronzi, D. (2016). The Impact of Children's Connection to Nature: Report for the Royal Society for the Protection of Birds (RSPB). University of Derby, 1-25. doi:10.13140/RG.2.1.3135.7206

Rozzi, R., Draguicevic, J. M., Arango, X., Sherriffs, M., Ippi, S., Anderson, C., . . . Massardo, F. (2005). Desde la ciencia hacia la conservación: el programa de educación y ética ambiental del Parque Etnobotánico Omora. Revista Ambiente y Desarrollo, 21(2), 2029.

Vallejos, M. (2012). La motivación, la actitud hacia las ciencias, la ansiedad y las estrategias metacognitivas de lectura en el rendimiento de los estudiantes universitarios: un análisis longitudinal (Tesis Doctoral). Madrid: Universidad Complutense de Madrid.

Vásquez, E., \& Wunderle, J. (2010). Differences in knowledge about birds and their conservation between rural and urban residents of Puerto Rico. Journal of Caribbean Ornithology, 23(2), 93-100. 